(C) 2019 IEEE. Personal use of this material is permitted. Permission from IEEE must be obtained for all other uses, in any current or future media, including reprinting/republishing this material for advertising or promotional purposes, creating new collective works, for resale or redistribution to servers or lists, or reuse of any copyrighted component of this work in other works. 


\title{
Electrically Small, Low Profile, Highly Efficient, Huygens Dipole Rectennas for Wirelessly Powering Internet-of-Things (IoT) Devices
}

\author{
Wei Lin, Member, IEEE, Richard W. Ziolkowski, Fellow, IEEE and Jianquan Huang
}

\begin{abstract}
Wireless power transfer (WPT) technologies are a major trend for emerging Internet-of-Things (IoT) applications. Because they negate the need for heavy, bulky batteries and can power multiple elements simultaneously, WPT systems enable very compact, ubiquitous IoT wireless devices. However, the realization of high performance, ultra-compact (electrically small) rectennas, i.e., the rectifying antennas that enable mid-range and far-field WPT, is challenging. We present the first electrically small $(k a<0.77)$ and low profile $(0.04 \lambda 0)$ linearly (LP) and circularly (CP) polarized WPT rectennas at $915 \mathrm{MHz}$ in the IMS band. They are facilitated by the seamless integration of highly efficient rectifiers, i.e., RF signal to DC power conversion circuits, with electrically small Huygens dipole $L P$ and $C P$ antennas. Their optimized prototypes have cardioid, broadside radiation patterns and effective capture areas larger than their physical size. Experimental results validate that they achieve an $89 \%$ peak $\mathrm{AC}$ to-DC conversion efficiency, effectively confirming that they are ideal candidates for many of the emerging IoT applications.
\end{abstract}

Index Terms - Electrically small rectennas, Huygens radiation pattern, internet-of-things (IoT), rectifier circuits, wireless power transfer.

\section{INTRODUCTION}

$\mathrm{T}$ he concept of wireless power transfer (WPT) - transferring power from one point to another separate point wirelessly can be traced back to Nikola Tesla and his experiments attempting to prove his conjectures at the turn of the 20th century [1], [2]. These non-radiative energy transference concepts have evolved into current near-field and short-range non-radiative-based WPT devices that recharge batteries in cell phones and EVs (electric vehicles) [3] - [6], as well as active implantable medical devices (AIMDs) and pacemakers [7], [8]. Aside from these near-field WPT applications, far-field microwave power transfer (MPT) enables long distance wireless power transmission. The first MPT system was demonstrated by W. C. Brown at Raytheon in 1963 [9]. These far-field radiative MPT systems continue to be investigated [10] $-[13]$.

Manuscript received on 11 November 2018, revised on 11 February 2019

Wei Lin and Richard. W. Ziolkowski are with the Global Big Data Technologies Centre, School of Electrical and Data Engineering, University of Technology Sydney, Ultimo NSW 2007, Australia, (E-mail: wei.lin@uts.edu.au).

Jianquan Huang is with the School of Electronics Information and Electrical Engineering, Xiangnan University, China.
The more recent pursuit of the Internet of Things (IoT) paradigms [14] has led to the intense development of a variety of far-field WPT technologies particular to the fifth generation $(5 \mathrm{G})$ of wireless systems [15]. It is expected that the associated ubiquitous wireless electronic devices have the potential to revolutionize many aspects of human life. They are being employed not only in consumer areas like communications, personal entertainment, smart homes, intelligent transportation systems, and smart cities, but also for security and health-care applications. However, the lack of compact, long-lasting portable power sources has hindered the deployment of many IoT concepts, e.g., current battery and supercapacitor systems are too large, too heavy, or too short-lived. Moreover, the next generation of sensors and control systems are embedded in the IoT electronic devices [16] - [19]. Consequently, any battery replacement will be difficult or even impractical. For these reasons, it is important to avoid the traditional battery as a power source and to develop new efficient and reliable ways to power devices wirelessly [20] in future IoT applications.

There are two main approaches to wirelessly powering devices remotely and robustly. One exploits already available ambient electromagnetic resources through wireless energy harvesting $(\mathrm{WEH})$, i.e., unintentional WPT, and the other is enabled by intentional WPT [21], [22]. WEH takes advantage of existing frequency bands [23] - [26] and requires broadband or multi-band antennas to capture as much of the available spectrum as is possible [27] - [34]. In general, the magnitudes of transferred wireless power in WEH applications are small amounts. On the other hand, intentional WPT applications aim to transfer as much power as possible from the wireless source to the targeted devices.

A major component of any far-field WPT system is a rectenna, i.e., a receiving antenna integrated with a rectifying circuit to convert the incident electromagnetic (AC) power into DC power. Narrowband operation in specific frequencies such as the (free) ISM bands is optimal. To capture larger amounts of power, large-scale rectenna arrays are usually implemented [35] - [39]. However, IoT devices such as RFID (radio frequency identification) tags, sensors, and communication devices require a single rectenna that is electrically small, but has a large energy capture capacity (e.g., cardioid-shaped radiation patterns to acquire the maximum amount of power from their driving sources while not wasting power radiated in 
the opposite direction) and high power conversion efficiency. Moreover, a circularly polarized (CP) system has notable advantages over a linearly polarized (LP) one in certain scenarios in which the orientation of the rectenna is not guaranteed and the WPT source is either a LP or CP system. Nonetheless, despite these highly desirable properties, no WPT rectenna has been reported to date that exhibits all of them.

In this paper, we introduce the first reported electrically small, highly efficient, Huygens linear (LP) and circular (CP) rectennas to date. Their designs are successfully facilitated by the seamless integration of significantly modified versions of our previously reported electrically small LP and CP Huygens dipole antennas [40], [41] and highly efficient AC-to-DC convertor circuits, i.e., rectifiers. Three innovations led to the successful Huygens LP (HLP) and CP (HCP) prototypes. First, a very compact and highly efficient rectifier circuit was developed. A combination of lumped components and a coplanar strip (CPS) line facilitated its seamless attachment to both electrically small Huygens radiators. Second, the HLP antenna was specifically redesigned to have its input impedance conjugate matched to the impedance of the rectifier, $68-\mathrm{j} 133 \Omega$, rather than to the $50 \Omega$ source. This removed the lossy inductor that was necessary for testing the rectifier circuit alone with the $50 \Omega$ source and, in turn, facilitated a significant enhancement of the maximum AC to DC conversion efficiency. Third, a CPS extension line was introduced for the HCP rectenna. This modification of the HLP design was critical; it prevented interference between the antenna and the rectifier.

The $915 \mathrm{MHz}$ ISM band (902-928 MHz, Region 2, Americas, Greenland, and eastern Pacific Islands) was selected for our optimized proof-of-concept prototypes. The experimental results for both the HLP and CP (HCP) prototype systems demonstrate the efficacy of their designs. They have cardioid, broadside radiation patterns and effective capture areas significantly larger than their physical size. Although being electrically small $(k a<0.77)$ and low profile $\left(0.04 \lambda_{0}\right)$ at $915 \mathrm{MHz}\left(\lambda_{0}=327.64 \mathrm{~mm}\right)$, they achieve an $89 \%$ peak AC-toDC power conversion efficiency. These experimental results confirm that they are ideal candidates for many of the emerging IoT devices and their applications.

\section{DESIGN COMPONENTS}

The methodology to realize an electrically small, highly efficient, Huygens WPT rectenna system is to seamlessly integrate an electrically small Huygens antenna and a highly efficient $\mathrm{AC}$ to DC convertor circuit together into a compact platform as illustrated in Fig. 1. The developed rectenna is able to capture wireless energy from broad incident angles and convert them into DC power with high efficiency.

\section{A. Electrically small Huygens antenna system}

A Huygens radiation pattern with high directivity is preferred in WPT applications for IoT devices. Its uni-directional cardioid-shaped form has a large (front to back ratio) FTBR value and broad beamwidths that are identical in all vertical planes. Fig. 1 illustrates the basic Huygens dipole system. An infinitesimal electric dipole (ED) is oriented along the $\mathrm{x}$-axis.
An infinitesimal magnetic dipole (MD) is oriented orthogonal to it along the -y-axis. The far-field expressions for their electric fields: $\vec{E}_{E D}$ and $\vec{E}_{M D}$ are [42], [43]:

$$
\begin{aligned}
\vec{E}_{E D}^{f f} & =j k \eta\left[I_{e} L\right] \cdot \frac{e^{-j k r}}{4 \pi r} \cdot[\hat{r} \times \hat{r} \times \hat{x}] \\
& =j k \eta\left[I_{e} L\right] \cdot \frac{e^{-j k r}}{4 \pi r} \cdot[-\cos \theta \cos \varphi \hat{\theta}+\sin \varphi \hat{\varphi}] \\
\vec{E}_{M D}^{f f} & =-j k\left[I_{m} L\right] \cdot \frac{e^{-j k r}}{4 \pi r} \cdot[\hat{r} \times(-\hat{y})] \\
& =j k\left[I_{m} L\right] \cdot \frac{e^{-j k r}}{4 \pi r} \cdot[-\cos \varphi \hat{\theta}+\cos \theta \sin \varphi \hat{\varphi}]
\end{aligned}
$$

where $I_{e} L$ is the electric dipole current moment; $I_{m} L$ is the magnetic dipole current moment; and $k=2 \pi / \lambda_{0}, \lambda_{0}$ being the free space wavelength corresponding to the operational frequency. With $I_{e}$ and $I_{m}$ being orthogonal and in-phase, the balanced condition: $\eta I_{e}=I_{m}$, yields the Huygens LP far-field radiation pattern:

$$
\begin{aligned}
\vec{E}_{E D}^{f f}+\vec{E}_{M D}^{f f}= & j k \eta\left[I_{e} L\right] \cdot \frac{e^{-j k r}}{4 \pi r} \\
& \cdot(1+\cos \theta) \cdot[-\cos \varphi \hat{\theta}+\sin \varphi \hat{\varphi}]
\end{aligned}
$$

The cardioid behavior is clearly attained, i.e., the maximum total field is twice that of either individual field in the broadside, $\theta=0^{\circ}$ direction and zero in the opposite, $\theta=180^{\circ}$ direction. Its 3D directivity pattern is illustrated in Fig. 1.

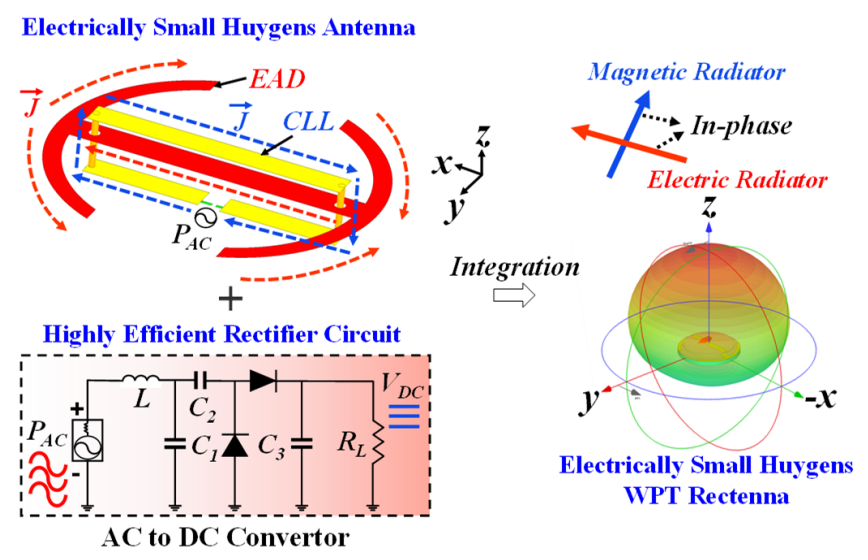

Fig. 1. Design concept of the electrically small, highly efficient, Huygens WPT rectenna system.

To realize electrically small Huygens antenna systems, metamaterial-inspired electrically small near-field resonant parasitic (NFRP) elements: the Egyptian Axe Dipole (EAD) and the capacitively-loaded loop (CLL), are integrated together into compact entities. Both LP and $\mathrm{CP}$ versions were investigated in our previous works [40], [41]. We successfully modified those designs to permit their seamless integration with the highly efficient rectifier circuit and to realize the first electrically small, highly efficient, Huygens WPT rectenna systems. As the power flow results given in Fig. 2 illustrate, the resulting rectenna strongly channels the incident field into it, making its effective area much larger than its electrically small physical area. This combination leads to a highly efficient AC- 
DC power conversion system that is ideal for many of the emerging IoT WPT applications.

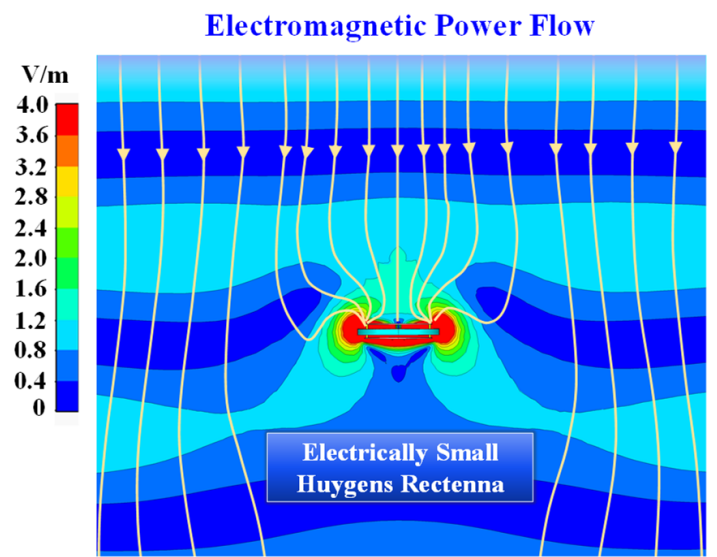

Fig. 2. Simulated electric field distribution and power flow under illumination by a normally incident LP plane wave. (Note the effective area of the HLP antenna is much larger than its physical cross section).

\section{B. Highly efficient rectifier (AC to DC convertor circuit)}

The essential conversion component of the desired far-field WPT system is a highly efficient rectifier, i.e., an AC to DC convertor circuit. It converts the received microwave power into dc power [44], [45]. As illustrated in Fig. 1, it is typically composed of a matching circuit, an input low-pass filter, a rectifying circuit, an output smoothing filter and a load resistor. An inductor, $L$, and two capacitors, $C_{1}$ and $C_{2}$, constitute a Ttype network that matches the input impedance of the rectifying circuit to the input impedance of the Huygens antenna. It should be noted that the inductor $L$ also acts as the input filter, suppressing re-radiation of the higher harmonics that are generated by the nonlinear, rectifying diode. Moreover, the capacitor $C_{2}$ also acts as an energy storage device. The vertical diode facilitates charging it during each negative portion of the sinusoidal signal. This stored energy is released during the positive portions, doubling the output voltage. A full wave rectifying circuit that consists of two Schottky diodes is adopted in this design to achieve the realized high output voltage and conversion efficiency. The capacitor $C_{3}$ on the output side of the rectifier serves as a smoothing filter, which minimizes the ripples in the DC voltage that is realized across the output port resistor $R_{L}$.

\section{HUYGENS LINEARLY-POLARIZED RECTENNA}

The design and operating principles of the electrically small LP Huygens WPT rectenna system are described. It is attained by seamlessly integrating the HLP antenna directly with the high efficiency rectifier. The fabricated prototypes of the rectifier and the entire WPT system are then presented along with the associated experimental results.

\section{A. System configuration and simulated results}

The HLP rectenna configuration is shown explicitly in Fig. 3. All of the components are fabricated on three (\#1 to \#3) Rogers $^{\text {TM }} 5880$ copper-cladded layers. Each layer is a circular disk substrate that is composed of a $0.5 \mathrm{oz}(17 \mu \mathrm{m})$ thick copper cladding of a dielectric whose relative permittivity and permeability equal to 2.2 and 1.0 , respectively, and whose loss tangent is 0.0009 . The thickness of substrate $\# 1$ and $\# 3$ is 0.7874 $\mathrm{mm}$ and of substrate $\# 2$ is $0.508 \mathrm{~mm}$. The top and bottom pieces of the metallic CLL element are etched, respectively, on the top surface of substrate\#1 (top disk) and on the top surface of substrate\#3 (bottom disk). They are connected by two copper posts that pass through and are normal to substrate\#2. The EAD element is etched on the top surface of the substrate\#2 (middle disk). The copper posts of the CLL pass through holes in the center arm of the EAD element to avoid any electrical connection to it and provide mechanical support of substrate\#3. The middle disk is supported by a Rohecell ${ }^{\mathrm{TM}}$ foam layer, which has a relative permittivity of 1.05 and essentially no loss. The short driven dipole and the rectifier circuit are located on the bottom surface of substrate\#3.

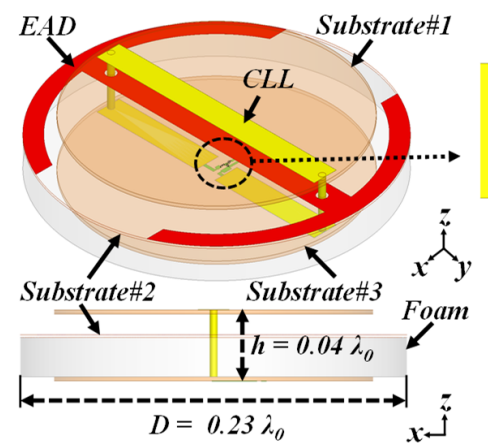

Rectifier Integration

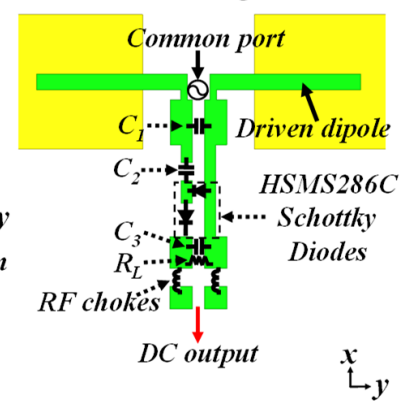

Fig. 3. Electrically small Huygens LP rectenna system configuration.

The HLP receiver and its rectifier load share a common port. As indicated in Fig, 3, the rectifier circuit consists of three capacitor components, along with the two Schottky diodes. The received AC power is converted into DC power at its output port resistor $R_{L}$. In order to eliminate any interference introduced by the DC output lines in the measurement setup, two RF chokes are added at the DC output port. It is emphasized that the standard input low-pass filter and the impedance matching inductor $L$ indicated in Fig. 1 are not present. To minimize the number of components and to increase the efficiency, they have been eliminated simply by tuning the HLP antenna to provide the appropriate inductive input impedance value. This approach was introduced in [46]; it avoids the nonnegligible loss of the lumped inductor (around 6\%) and compensation techniques adopted, for instance, in [47]. The entire structure is electrically small $(k a<0.73)$ and low profile $\left(0.04 \lambda_{0}\right)$ at its resonance frequency $f_{0}=915 \mathrm{MHz}$, where the corresponding free space wavelength $\lambda_{0}=327.64 \mathrm{~mm}$, the wave number $k=2 \pi / \lambda_{0}$, and $a$ is the radius of the smallest sphere enclosing it. It is also compact transversely with only a $0.115 \lambda_{0}$ $=37.68 \mathrm{~mm}$ radius.

The current distributions on the orthogonally-oriented electric and magnetic dipoles of this electrically small HLP antenna are depicted in Fig. 4. The major currents are concentrated on the CLL element at the times 0 and 2T/4, where $\mathrm{T}$ is one period of the source excitation. They are concentrated 
on the EAD element at the times T/4 and 3T/4. Since the CLL represents a current loop of area $A$ that is driven with essentially a constant current $I_{0}$ in free-space, its magnetic current moment $I_{m}=j \omega \mu_{0} I_{0} A / L$, where $L$ is the inductance of the loop [48]. Thus, the phase of the magnetic dipole is $90^{\circ}$ ahead of the actual loop current. Consequently, as shown, these " $E$ " and " $M$ " dipoles are resonating in-phase as required to achieve the desired broadside cardioid antenna pattern.

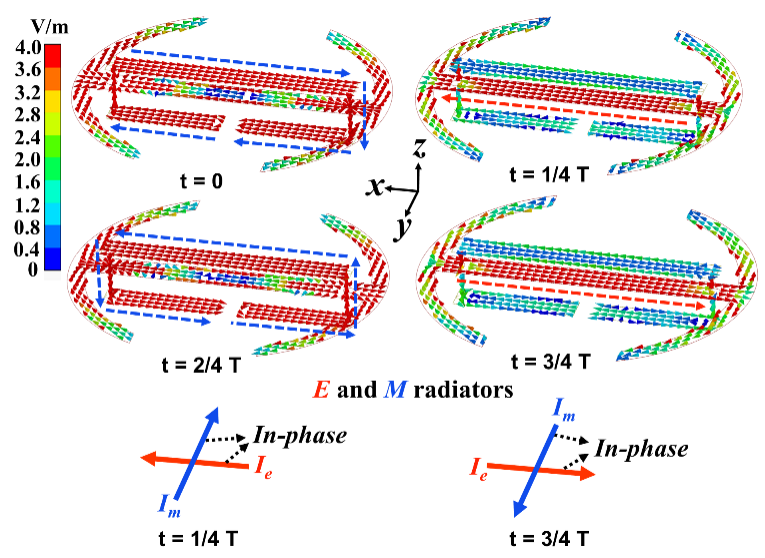

Fig. 4. The current distributions on the electric and magnetic NFRP elements over one period $\mathrm{T}$ of the source frequency demonstrate their in-phase behavior.

The simulated radiation performance characteristics of the optimized HLP rectenna are given in Fig. 5. Unlike the design in [41] which matches to $50 \Omega$ source, the input impedance of this HLP antenna at $915 \mathrm{MHz}$ is $68+\mathrm{j} 133 \Omega$, which is completely conjugate matched to the impedance of the rectifier circuit, notably providing the requisite inductance to compensate for its capacitance. Its cardioid-shaped $2 \mathrm{D}$ realized gain patterns are almost identical in the two vertical planes $\varphi=$ $0^{\circ}$ and $\varphi=90^{\circ}$. They have identical very broad $3 \mathrm{~dB}$ beamwidths, $\pm 67^{\circ}$, about the broadside direction in the upper hemisphere. The broadside realized gain is $3.8 \mathrm{dBi}$. The simulated radiation efficiency is $84 \%$.

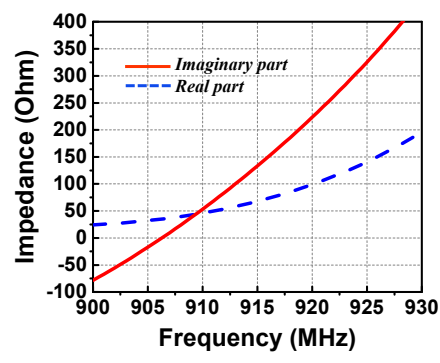

(a)

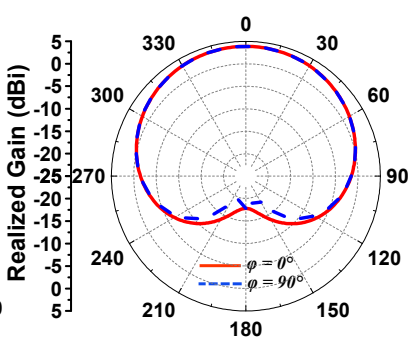

(b)
Fig. 5. Simulated HLP rectenna performance. (a) Input impedance as a function of the source frequency. (b) $2 \mathrm{D}$ realized gain patterns at $915 \mathrm{MHz}$.

\section{B. Measured results of the rectifier circuit alone}

To validate the performance of the rectifier circuit, it was fabricated, assembled and tested alone. To replace the antenna in this configuration, it was fed by an input $50 \Omega$ differential source via a balun as shown in Figs. 6(a) and 6(b). A Coilcraft ${ }^{\mathrm{TM}}$ lumped inductor $L$ was selected for this matching circuit. To achieve the desired high AC-to-DC conversion efficiency,
HSMS286C Schottky diodes from Broadcom ${ }^{\mathrm{TM}}$ were selected. The package information can be found from [49]. Murata ${ }^{\mathrm{TM}}$ capacitors were selected for the corresponding $C_{1}$ to $C_{3}$ lumped elements. The resistor $R_{L}$ is from Yageo ${ }^{\mathrm{TM}}$. The two RF chokes were $560 \mathrm{nH}$ inductors from Murata ${ }^{\mathrm{TM}}$ (yielding isolation larger than $30 \mathrm{~dB}$ from 900 to $930 \mathrm{MHz}$ ). All of these lumped components were in 0403 (1 mm length) surface-mount device (SMD) packages. The rectifier circuit was highly compact. Its total length, including all the components, was only $7.3 \mathrm{~mm}$ (0.02 $\lambda$ ). Compared with the previously developed rectifier designs that were cascaded to dipole antennas, e.g., [50], [51], the entire size of our HLP system is electrically small. The lengths of the rectifiers in [50] and [51] are larger than $0.5 \lambda_{0}$ and $0.15 \lambda_{0}$, respectively. In fact, those circuits cannot even be considered for our system since it has a radius of only $0.115 \lambda_{0}$.

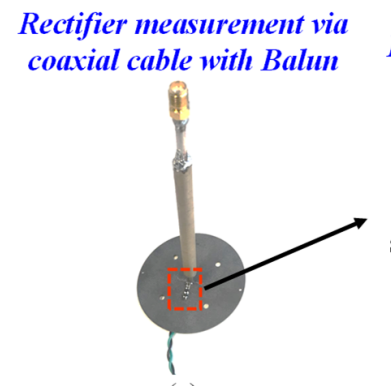

(a)

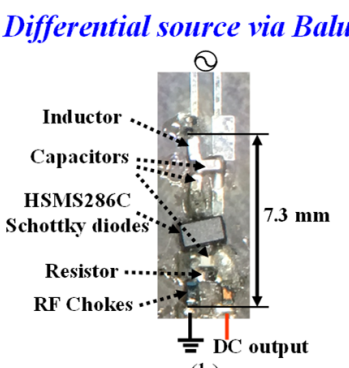

(b)

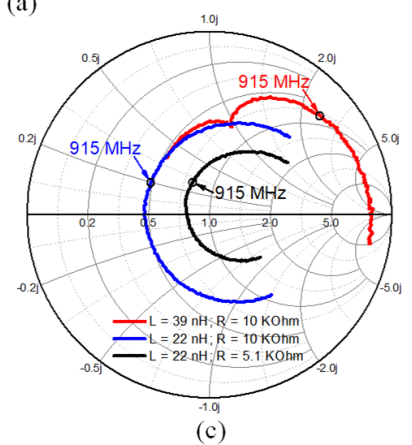

Fig. 6. Rectifier circuit alone measurements. (a) Photo of rectifier under test. (b) Enlarged view of the rectifier circuit with differential excitation. (c) Measured input impedance matching results for different choke and output resistor values. Input power: $5 \mathrm{dBm}(3.16 \mathrm{~mW})$.

Due to a discrepancy arising between the actual parameters of the HSMS286C diodes and those in the ADS (Advanced Design System from Keysight Technologies ${ }^{\mathrm{TM}}$ ) simulation model, the actual values of the components in the matching circuit and the load had to be tuned manually to achieve an optimized measured performance. The optimized simulation model component values were: $L=39 \mathrm{nH} ; C_{1}=0.4 \mathrm{pF} ; C_{2}=$ $100 \mathrm{pF} ; C_{3}=100 \mathrm{pF} ; R_{L}=10 \mathrm{~K} \Omega$. These values provided the initial values tried in the experiments. However, as shown with the Smith Chart in Fig. 6(c), the measured input impedance had a high inductance at $915 \mathrm{MHz}$. To compensate for this fact and to achieve good impedance matching, the initial $39 \mathrm{nH}$ inductor and $10 \mathrm{~K} \Omega$ load resistor were replaced by an available $22 \mathrm{nH}$ inductor and a $5.1 \mathrm{~K} \Omega$ resistor.

After this element tuning, good impedance matching was achieved as shown in Fig. 7(a). The $\left|S_{11}\right|$ values were below -10 $\mathrm{dB}$ from 825 to $945 \mathrm{MHz}$ for a variety of input power levels. 
The bandwidth is wide and covers the targeted $915 \mathrm{MHz}$ ISM band. Fig. 7(b) presents the measured and simulated AC-to-DC conversion efficiency (output DC power divided by the input $\mathrm{RF} / \mathrm{AC}$ power) as functions of the input power at $915 \mathrm{MHz}$. The rectifier reaches its peak efficiency of $80.3 \%$ at $10 \mathrm{dBm}$ input power, i.e., the DC output power is $8.03 \mathrm{~mW}$ when the input power is $10 \mathrm{~mW}$. In addition, the AC-to-DC conversion efficiency is larger than $50 \%$ when the input power is ranges widely from $-5 \mathrm{dBm}(0.3 \mathrm{~mW})$ to $13.5 \mathrm{dBm}(22.4 \mathrm{~mW})$. Consequently, the measured results confirm, in agreement with their simulated values, that the developed rectifier circuit facilitates a high AC-to-DC conversion efficiency.

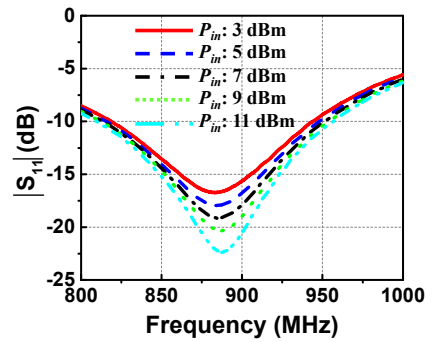

(a)

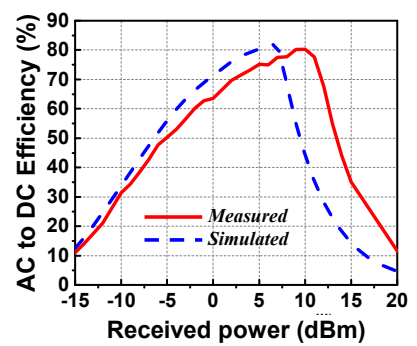

(b)
Fig. 7. (a) Measured $\left|\mathrm{S}_{11}\right|$ of the rectifier circuit as functions of the source frequency for different input powers. (b) Measured and simulated AC to DC conversion efficiency as functions of the input power at $915 \mathrm{MHz}$.
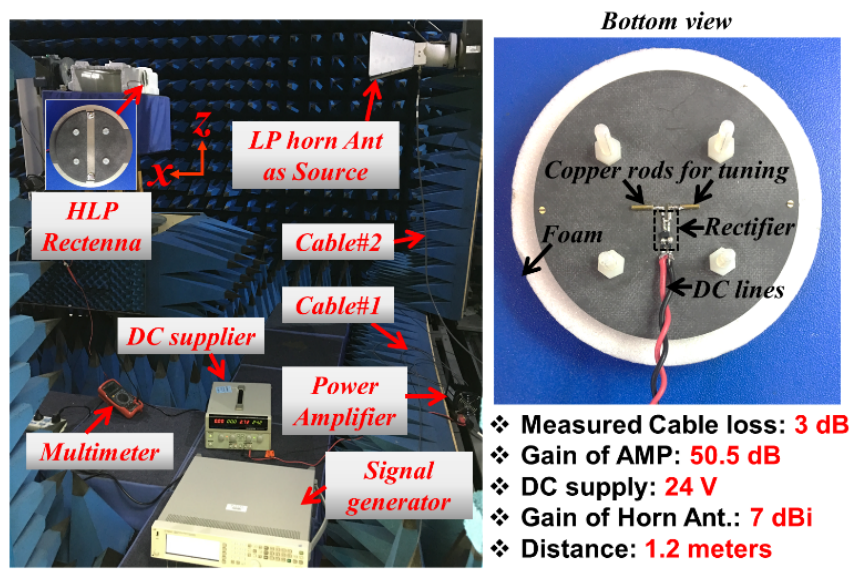

Fig. 8. Measurement setup and the fabricated electrically small HLP rectenna.

\section{HLP rectenna measured results}

To validate the performance of the HLP rectenna, its prototype was fabricated and measured in the far field anechoic chamber environment shown in Fig. 8. The measurement setup shown in Fig, 8(a) consisted of a rectangular horn antenna, a power amplifier, two RF cables, a signal generator, a DC power supplier, and a multimeter. The electromagnetic source was a linearly- polarized, wideband, double ridge rectangular horn antenna. Its polarization was fixed in the setup; it was oriented along the vertical direction as shown in the photo. Its measured realized gain at $915 \mathrm{MHz}$ is $7.0 \mathrm{dBi}$. The power amplifier was a Mini-Circuits ${ }^{\mathrm{TM}} \mathrm{ZHL}-20 \mathrm{~W}-13 \mathrm{SW}+[52]$; it had a gain of 50.5 dB. The signal generator was from Keysight Technologies ${ }^{\mathrm{TM}}$. Cable\#1 connected the output port of the signal generator and the input port of the power amplifier. Cable\#2 connected the output port of the power amplifier and the input port of the horn antenna. The total measured loss from the two cables around $915 \mathrm{MHz}$ was $3 \mathrm{~dB}$. A DC voltage supply provided $24 \mathrm{~V}$ to the power amplifier. The multimeter measured the DC voltage output from each rectenna through two DC lines located after the two RF chokes in the rectifier circuit. The optimized electrically small HLP rectenna was fabricated as shown in Fig. 8 (b). The rectifier is seen to be seamlessly integrated with the antenna. Two DC lines were connected to the DC output of the rectifier for ease of the measurements. Two short, thin $(0.8 \mathrm{~mm}$ diameter) copper rods were attached on the short driven dipole for tuning purposes.

Fig. 9 presents the measured output DC voltage and AC-toDC conversion efficiency of the electrically small HLP rectenna as functions of the received power at $907 \mathrm{MHz}$, the frequency at which the maximum output DC voltage occurred. It is a mere $0.87 \%$ shift $(8 \mathrm{MHz}$ ) from the designed $915 \mathrm{MHz}$ resonance frequency and still within the ISM band as shown in Fig. 10(b). To evaluate the conversion efficiency, we employed the standard approach which is clearly discussed in [16] and has been used for rectenna reports throughout the literature as evidenced, e.g., in [17] - [39].

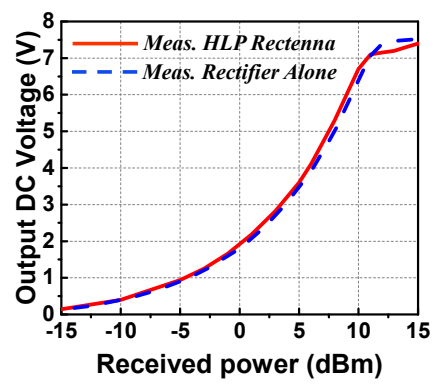

(a)

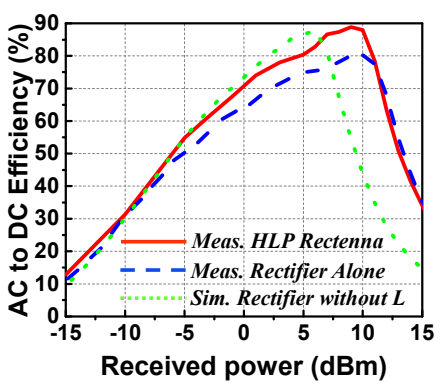

(b)
Fig. 9. Electrically small HLP rectenna and the rectifier alone. (a) Measured output DC voltage as functions of the received power at $907 \mathrm{MHz}$. (b) Measured and simulated $\mathrm{AC}$ to DC conversion efficiency at $907 \mathrm{MHz}$.

The amount of power delivered to the rectenna is calculated according the Friis transmission equation [42]:

$$
\frac{P_{r}}{P_{t}}=G_{t} \times G_{r} \times\left(\frac{\lambda_{0}}{4 \pi R}\right)^{2}
$$

where $P_{r}$ is the power received by the rectifier; $P_{t}$ is the transmitted power measured from the source horn antenna; $G_{t}$ is the gain of the horn antenna $(7 \mathrm{dBi}) ; G_{r}$ is the gain of the Huygens radiator $(3.8 \mathrm{dBi}) ; \lambda_{0}$ is the wavelength at $907 \mathrm{MHz}$ (330 mm); and $R$ is the distance between the horn antenna and the rectenna $(1.2 \mathrm{~m})$. The maximum linear dimension, $\mathrm{D}$, of the horn antenna was $0.3 \mathrm{~m}$ and the targeted operational frequency of the rectennas was $915 \mathrm{MHz}$. Consequently, the corresponding free space wavelength was $\lambda_{0}=0.328 \mathrm{~m}$ and the far field of the horn antenna was reached at the distance $2 \mathrm{D}^{2} /$ $\lambda_{0}=0.55$ meters away from it. Consequently, the rectenna under test at 1.2 meters away from the source antenna was well into its far field.

Fig. 9(a) compares the measured output DC voltage values 
of the HLP rectenna and the rectifier circuit alone as functions of the received power. Their trends are in good agreement. The output DC voltage rapidly increases after the received power is $0 \mathrm{dBm}(1 \mathrm{~mW})$ and saturates at $10 \mathrm{dBm}(10 \mathrm{~mW})$ to the value of 7.4 V. Fig. 9(b) compares the measured and simuated ACto-DC conversion efficiencies of the HLP rectenna and the rectifier alone. Their trends agree very well. Because its design eliminated the lossy lumped inductor, the HLP rectenna achieves higher efficiencies than the rectifier alone. The measured maximum AC-to-DC efficiency of the HLP rectenna reaches $88.9 \%$ when the received power is $9.0 \mathrm{dBm}$. The corresponding maximum of the rectifier alone is only $80.3 \%$ when the received power is $10 \mathrm{dBm}$. Furthermore, Fig. 9(b) shows the simulated maximum AC to DC conversion efficiency without that inductor being present is $87.8 \%$. Both of these results confirm the negative impact that the loss of the lumped inductor has on the rectifier's conversion efficiency and emphasize the advantage that was accomplished by directly matching the antenna to the rectifying circuit. It is also noted from Fig. 9(b) that the conversion efficiency is greater than $50 \%$ over a wide range of input powers, i.e., from $-6 \mathrm{dBm}(250 \mu \mathrm{W})$ to $13 \mathrm{dBm}(20 \mathrm{~mW})$. These measurements clearly demonstrate the high conversion efficiency of the HLP rectenna.

In addition, another set of measurements was carried out as the rectenna was rotated in relation to the horn's LP direction to highlight its wide-angle power capture. The measured DC voltages at $907 \mathrm{MHz}$ were 5.7, 5.0, and 4.3V when the HLP rectenna was rotated clockwise around the z-axis (as observed from its top) by $30^{\circ}, 45^{\circ}$, and $60^{\circ}$, respectively. The measured DC voltages were $5.6,4.9$, and $4.2 \mathrm{~V}$ when it was rotated in the anti-clockwise direction by $30^{\circ}, 45^{\circ}$, and $60^{\circ}$, respectively. These measured symmetrical results confirm the ability of the HLP rectenna to receive power over large angles of incidence in agreement with the wide beamwidths of the simulated patterns in Fig. 5(b), as well as its large effective capture area represented by the power flow curves illustrated in Fig. 2.

\section{HUYGENS CIRCULARLY-POLARIZED RECTENNA}

The HLP rectenna is highly suited to WPT situations in which it can be aligned preferentially to an LP source. On the other hand, there is a polarization mismatch issue if it cannot be. Polarization mismatch can have significant ramifications on the performance of any type of one-way or two-way electromagnetic (EM) system. The top illustration in Fig. 10 clearly shows the configuration in which the electric field of the incident EM wave generated by the source is oriented perpendicular to the LP direction of the rectenna, i.e., perpendicular to the center conductor of the EAD NFRP element. This incident EM field will thus pass through the rectenna without any significant interactions with it as the simulated power flow results in Fig. 10(a) depict. The measured output DC voltage values as a function of the source frequency when the microwave power source was polarization aligned and mismatched with the HLP rectenna are shown in Fig. 10(b). Clearly, there was essentially no DC signal detected in the mismatch configuration. This polarization mismatch issue focuses attention on the need for $\mathrm{CP}$ systems to mitigate its impact.

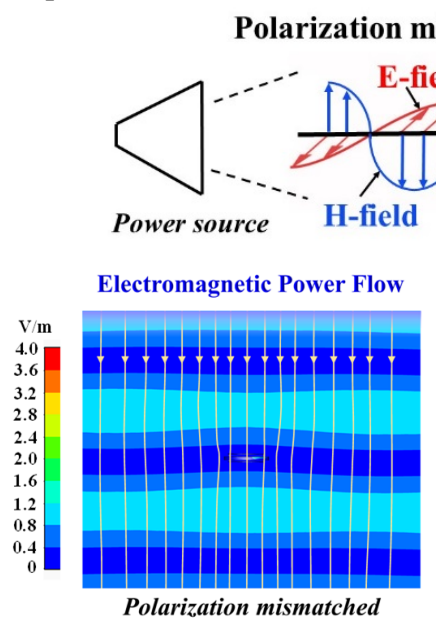

(a)
Fig. 10. Demonstration of the polarization purity of the electrically small HLP rectenna. (a) Simulated power flow when the incident LP electromagnetic wave is orthogonal to it. (b) Measured DC output voltage as a function of the source frequency when the source and rectenna polarizations are matched and not.

\section{A. System configuration}

The HCP rectenna follows naturally from the HLP design. The HCP configuration, as shown in Fig. 11, combines two orthogonal pairs of EAD and CLL elements. The substrates and foam are identical to those in the HLP prototype. The arcs of the EAD are bent to achieve the requisite reactance in a more compact structure.

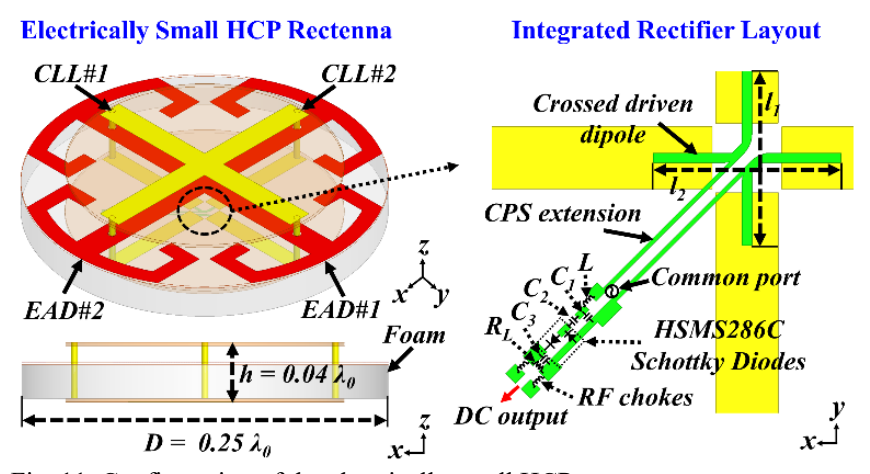

Fig. 11. Configuration of the electrically small HCP rectenna.

The major change is the "driven" dipole. An unbalanced crossed dipole pair was adopted and matched to the four NFPR elements for the HCP design. Circular polarization is produced if the lengths of these orthogonal dipoles, $l_{2}$ along the $x$-axis and $l_{l}$ along the $y$-axis, are different and properly adjusted to bring about the necessary $90^{\circ}$ phase difference. The length $l_{1}$ is 12.6 $\mathrm{mm}$ and $l_{2}$ is $13.5 \mathrm{~mm}$. The current distributions on the NFRP elements presented in Fig. 12 clearly demonstrate this behavior. In a period of time T, the orthogonal $E$ and $M$ elements are both rotating in a clockwise manner. Thus, a left-handed CP (LHCP) Huygens dipole directivity pattern is generated in this design.

The rectifier circuit is essentially the same as its LP design, except that the inductor $L$ is reintroduced in this CP design. It 
was very challenging to achieve an inductive port impedance and good CP radiation simultaneously. Both the input impedance and AR values change dramatically when the driven crossed dipole of the HCP antenna is adjusted. Because the input impedance of the $\mathrm{HCP}$ antenna at $915 \mathrm{MHz}$ was determined to be $42-\mathrm{j} 12 \Omega$, it was consequently decided to match the system directly to the $50 \Omega$ source without further modifications. Moreover, the rectifier is not directly attached to the driven dipole, but is positioned away from its center through an extended co-planar stripline (CPS). This configuration was necessary to prevent strong coupling between the rectifying circuit and the NFRP elements.

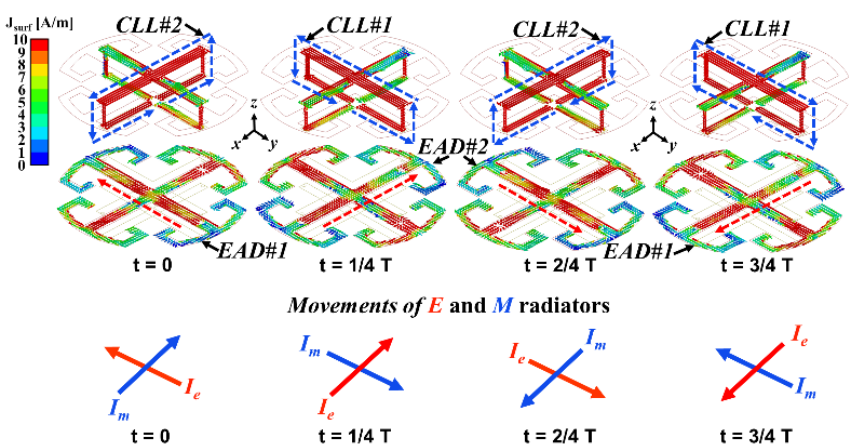

Fig. 12. Current density distributions on the crossed EAD and CLL NFRP elements over one period $\mathrm{T}$ of the source frequency and the corresponding orientations of the $E$ and $M$ dipoles illustrate the resulting LHCP behavior.

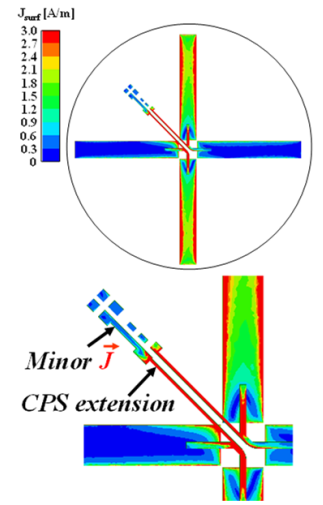

(a)

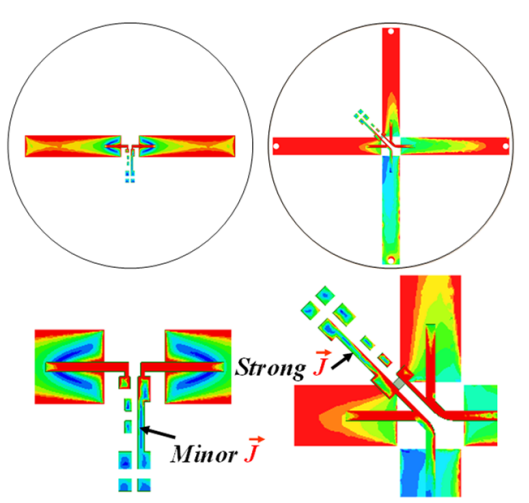

(c)
Fig. 13. Demonstration of the effects associated with the CPS extension to move the rectifying circuit away from the center of the dipole element. (a) HCP antenna with CPS feed extension. (b) HLP antenna without the CPS feed extension. (c) HCP antenna without the CPS feed extension.

\section{B. Key design considerations and simulated results}

The co-planar stripline (CPS) extension from the center of the "driven" dipole element of the HCP antenna to the input port of the rectifier is a critical design feature in the HCP rectenna system. As illustrated in Fig. 13, the metallic parts of the rectifier circuit impact the electromagnetic performance of the HCP antenna if they are too close to the center of the dipole. It is clearly observed in Fig. 13(a) that only minor currents are coupled to the rectifier if it is moved away from the dipole center and, hence, do not affect the system's CP characteristics. Similarly, Fig. 13(b) demonstrates that the currents coupled to the metallic parts of the rectifier are truly minor in the corresponding HLP system. This feature is expected because, in fact, the rectifier itself is orthogonal to the dipole. On the other hand, as observed in Fig. 13(c), strong currents on the rectifier are established from its coupling to the dipole element and the NFRP elements when it is too close to the center of the dipole. They significantly deteriorate the $\mathrm{CP}$ performance.

The impact of the CPS extension on the HCP rectenna is demonstrated further with the simulated $\left|S_{11}\right|$ and $A R$ values given in Fig. 14 as functions of the source frequency for the system with and without the extension. The overall length of the extended CPS line is $14 \mathrm{~mm}\left(\lambda_{0} / 23.4\right)$. The values $f_{l}$ and $f_{2}$ are the resonance frequencies of the two orthogonal sets of NFRP elements. The resonances nicely overlap only when the extension is present. As shown in Fig. 14(b), the operating frequency of the best CP performance falls at $915 \mathrm{MHz}$, which lies between $f_{1}$ to $f_{2}$. It is the frequency at which the phase difference between the resonant pairs of NFRP elements reaches $90^{\circ}$. On the other hand, both the impedance matching and the AR values also become quite poor when the rectifier is attached directly to the center of the dipole. These results clearly demonstrate the need for the CPS extension to move the rectifier circuit far enough away from the center of the dipole to eliminate the effects of their coupling.

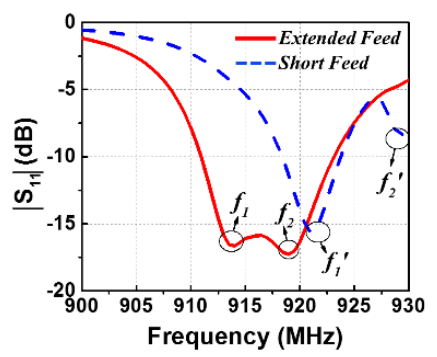

(a)

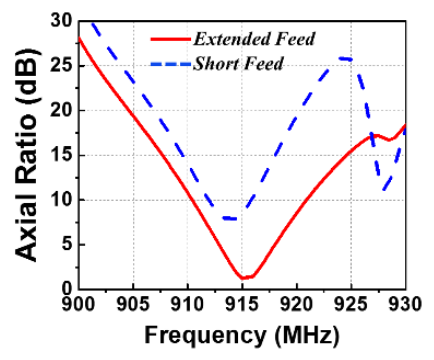

(b)
Fig. 14. Electrically small HCP rectenna. Simulated (a) $\left|S_{11}\right|$ and (b) AR values as functions of the source frequency.

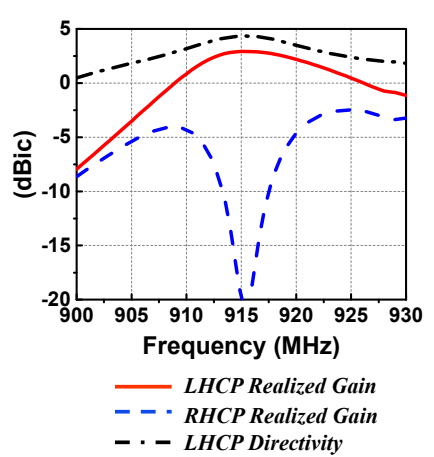

(a)

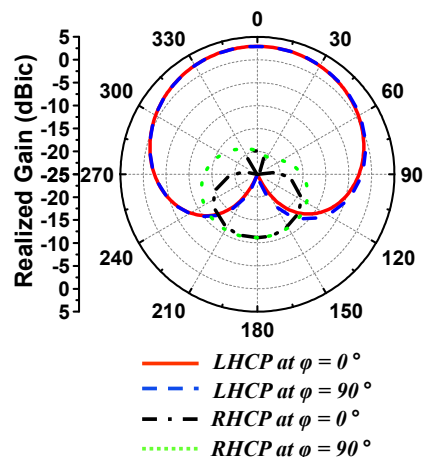

(b)
Fig. 15. Electrically small HCP rectenna. (a) Realized gain and directivity values as functions of the source frequency. (b) 2D realized gain patterns at 915 $\mathrm{MHz}$.

The simulated realized gain and directivity values as functions of the source frequency are shown in Fig. 15 (a). The simulated radiation efficiency is $74 \%$. The realized LHCP gain at $915 \mathrm{MHz}$ is $2.91 \mathrm{dBic}$ and the directivity is $4.35 \mathrm{dBic}$. The difference is $1.44 \mathrm{~dB}$, which is higher than the $0.86 \mathrm{~dB}$ value in the HLP case. Nonetheless, the increase is expected because the 
fields in the substrate and the current densities on the NFRP elements are larger than those in the HLP system. Fig. 15(b) gives the $2 \mathrm{D}$ realized gain patterns. The cardioid pattern is clearly attained; the cross-pol levels are quite low.

\section{HCP rectenna measured results}

The HCP rectenna was fabricated and tested. The prototype is shown in Fig. 16 along with the simulated power flow and measured output voltage and conversion efficiency results. Because the source is the same LP horn and the rectenna is now $\mathrm{CP}$, the rectenna will convert only half of the power incident on it. Consequently, the incident power level was increased by 3 $\mathrm{dB}$ (factor of 2). Fig. 16(a) illustrates again that the effective area of the HCP rectenna is much larger than its physical size. Moreover, it is found that the power flow behavior is the same when the rectenna is rotated about its broadside direction. In fact, similar DC output voltages are realized around $911 \mathrm{MHz}$ during this process. In particular, when it is rotated clockwise in steps of $45^{\circ}$ with respect to its broadside direction ( + z-axis), they are $5.5,4.7,4.7$, and $5.5 \mathrm{~V}$ for $0^{\circ}, 45^{\circ}, 90^{\circ}$ and $135^{\circ}$, respectively, as shown in Fig. 16(b). The received power difference between $5.5 \mathrm{~V}$ and $4.7 \mathrm{~V}$ is $1.2 \mathrm{~dB}$, as predicted by the corresponding simulations. Decent $\mathrm{CP}$ radiation receiving capacity is observed. The measured DC output voltages for the HLP and HCP systems are compared in Fig. 16(c). The trend of HCP system clearly performs almost identically to the HLP one.

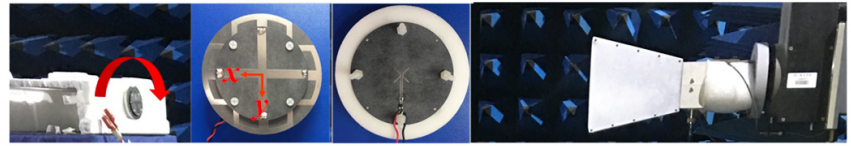

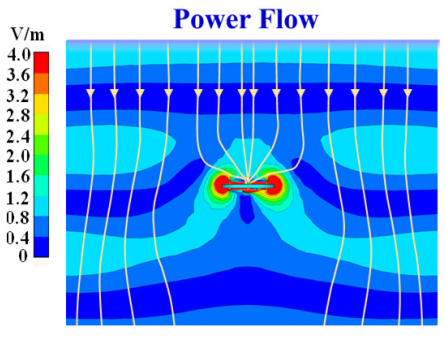

(a)

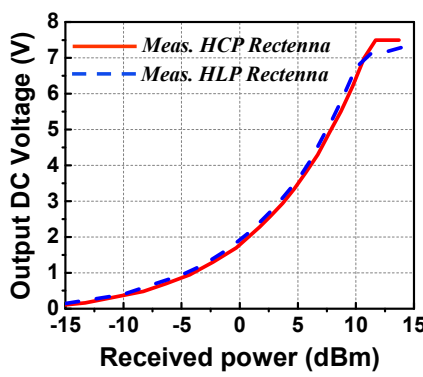

(c)

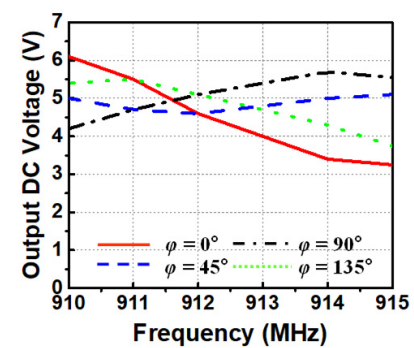

(b)

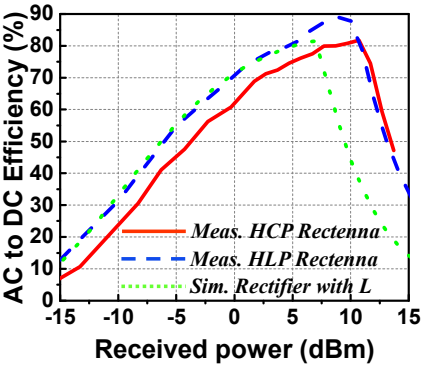

(d)
Fig. 16. Comparisons of the electrically small HCP and HLP rectennas. (a) Simulated electric field distribution and power flow into the HCP rectenna under illumination by a normally incident $\mathrm{CP}$ plane wave. (b) Measured output DC voltage as a function of the source frequency at different rotation angles. Comparisons of the measured (c) output DC voltages and (d) measured and simulated AC-to-DC conversion efficiencies at $911 \mathrm{MHz}$.
In the same manner, we also measured the output DC voltages at $911 \mathrm{MHz}$ when the HCP rectenna system was rotated about the EAD centerline along the $y$-axis. They were 4.6, 4.1, and 3.6 V for clockwise rotation (as observed from top) by $30^{\circ}, 45^{\circ}$, and $60^{\circ}$, respectively. The values were $4.7,3.9$, and $3.3 \mathrm{~V}$ when it was rotated in the counter-clockwise direction by $30^{\circ}, 45^{\circ}$ and $60^{\circ}$, respectively. These results help confirm the capacity of the rectennas to capture incident EM waves over a wide set of incidence angles. They confirm the very broad beamwidths of the HCP rectenna.

The power conversion efficiencies of the HCP and HLP rectennas are compared in Fig. 16(d). Again, since we used the LP horn as the power source, we used the equivalent LP gain of the rectenna to calculate the received power with (3) and, hence, the AC-to-DC conversion efficiency. The HCP measured values are slightly lower than the HLP ones. The peak value is $82 \%(9.6 \mathrm{~mW})$ when the received power is $10.7 \mathrm{dBm}(11.75$ $\mathrm{mW}$ ). As explained above, the decrease (from $88.9 \%$ to $82 \%$ ) in efficiency is caused by the presence of the lossy inductor $L$ in the rectifier. Unfortunately, as the inductor $L$ was present in this HCP rectenna because it was found to be quite difficult to align both the minimums of the reflection coefficient and AR values since they change simultaneously. The simulated results for the rectifier with the inductor $L$ alone also confirm this efficiency drop. The maximum simulated efficiency of the HCP rectenna is $82.2 \%$, which is very close to the measured efficiency, $82.0 \%$.

\section{CONCLUSION}

Huygens LP and CP rectenna systems with high AC-to-DC conversion efficiencies were presented for operation in the IMS band around $915 \mathrm{MHz}$. Both the HLP and HCP systems were low profile $\left(0.04 \lambda_{0}\right)$ and electrically small $(k a<0.73$ for the LP system and $k a<0.77$ for the CP version). The HLP system achieved a high $88.9 \%$ peak conversion efficiency. It is suitable for applications in which the polarization of the LP power source and its polarization are easily aligned. The HCP peak conversion efficiency was $82 \%$. It is suitable for applications in which the LP source cannot be aligned to the polarization of the HLP and is ideally suited for WPT applications when the source is also a CP device. Power flow simulations clearly illustrated that both devices have effective (power capture) areas much larger than their physical areas. Moreover, they both have large $3 \mathrm{~dB}$ beamwidths, $\pm 67^{\circ}$, about their broadside directions. The measured results affirm that both systems are excellent candidates for many emerging wireless IoT/IoE applications.

\section{ACKNOWLEDGEMENTS}

The authors would like to thank Prof. Y. Jay Guo, University of Technology Sydney, for his support of these efforts and Prof. Zheng Li from Beijing Jiao Tong University for his assistance in the measurement campaign. 


\section{REFERENCES}

[1] N. Tesla, “Apparatus for transmitting electrical energy," U.S. Patent 1119 732, Dec. 1914

[2] N. Tesla, "The true wireless", Electrical Experimenter, May 1919.

[3] A. Kurs, A. Karalis, R. Moffatt, J. D. Joannopoulos, P. Fisher, and M. Soljačić, "Wireless power transfer via strongly coupled magnetic resonances," Science, vol. 317, no. 5834, pp. 83-86, Jul. 2007.

[4] J. Shin, et al., "Design and implementation of shaped magneticresonance-based wireless power transfer system for roadway-powered moving electric vehicles," IEEE Trans. Industrial Electron., vol. 61, no. 3, pp. 1179-1192, Mar. 2014

[5] Z. Liu, Z. Chen, J. Li, and H. Zhao, "A shape-reconfigurable modularized wireless power transfer array system for multipurpose wireless charging applications," IEEE Trans. Antennas Propag., vol. 66, no. 8, pp. 4252 4259, Aug. 2018.

[6] C. C. Mi, G. Buja, S. Y. Choi, and C. T. Rim, "Modern advances in wireless power transfer systems for roadway powered electric vehicles," IEEE Trans. Industrial Electron., vol. 63, no. 10, pp. 6533-6545, Oct. 2016

[7] T. Campi, S. Cruciani, F. Palandrani, V. De Santis, A. Hirata, and M. Feliziani, "Wireless power transfer charging system for AIMDs and pacemakers," IEEE Trans. Microw. Theory Techn., vol. 64, no. 2, pp. 633642, Feb. 2016.

[8] L. Li, H. Liu, H. Zhang, and W. Xue, "Efficient wireless power transfer system integrating with metasurface for biological applications," IEEE Trans. Industrial Electron., vol. 65, no. 4, pp. 3230-3239, Apr. 2018.

[9] W. C. Brown, "The history of power transmission by radio waves," IEEE Trans. Microw. Theory Techn., vol. MTT-32, no. 9, pp. 1230-1242, Sep. 1964

[10] H. Matsumoto, "Research on solar power satellites and microwave power transmission in Japan,” IEEE Microw. Mag., vol. 3, no. 4, pp. 36-45, Dec. 2002.

[11] N. Shinohara, "Beam control technologies with a high-efficiency phased array for microwave power transmission in Japan," Proc. IEEE, vol. 101 no. 6 , pp. 1448-1463, Jun. 2013

[12] N. B. Carvalho, et al., "Wireless power transmission: R\&D activities within Europe,” IEEE Trans. Microw. Theory Techn., vol. 62, no. 4, pp. 1031-1045, Apr. 2014

[13] R. M. Dickson, "Power in the sky: Requirements for microwave wireless power beamers for powering high-altitude platforms," IEEE Microw. Mag., vol. 14, pp. 36-47, Apr. 2013.

[14] L. Roselli, et al., "WPT related applications enabling internet of things evolution," in Proc. 2016 10th European Conference on Antennas and Propagation (EuCAP 2016), 10-15 April 2016, Davos, Switzerland, pp. 1 -2 .

[15] M. R. Palattella, et al., "Internet of things in the 5G era: Enablers, architecture, and business models," IEEE J. Sel. Area. Commun., vol. 34 no. 3, pp. 510-527, Mar. 2016

[16] Z. Popović, E. A. Falkenstein, D. Costinett, and R. Zane, "Low-power farfield wireless powering for wireless sensors," Proc. IEEE, vol. 101, no. 6 , pp. 1397-1409, Jun. 2013

[17] H. J. Visser and R. J. M. Vullers, "RF energy harvesting and transport for wireless sensor network applications: Principles and requirements," Proc. IEEE, vol. 101, no. 6, pp. 1410-1423, Jun. 2013.

[18] S. Kim, et al., "Ambient RF energy-harvesting technologies for selfsustainable standalone wireless sensor platforms," Proc. IEEE, vol. 102 , no. 11, pp. 1649-1666, Nov. 2014

[19] O. Bjorkqvist, O. Dahlberg, G. Silver, C. Kolitsidas, O. Quevedo-Teruel, and B.L.G. Jonsson, "Wireless sensor network utilizing radio-frequency energy harvesting for smart building applications," IEEE Antennas Propag. Mag., vol. 60, no. 5, pp. 124 -136, Oct. 2018.

[20] A. Costanzo and D. Masotti, "Energizing 5G: Near- and far-field wireless energy and data transfer as an enabling technology for the 5G IoT," IEEE Microw. Mag., vol. 18, no. 3, pp. 125-136, May 2017.

[21] C. Liu, Y. X. Guo, H. Sun, and S. Xiao, "Design and safety consideration of an implantable rectenna for far-field wireless power transfer," IEEE Trans. Antennas Propag., vol. 62, no. 11, pp. 5798-5806, Nov. 2014.

[22] Z. Popovic, "Cut the cord: Low-power far-field wireless powering," IEEE Microw. Mag., vol. 14, no. 2, pp. 55-62, Mar. 2013.

[23] Z. Gu, S. Hemour, L. Guo, and K. Wu, "Integrated cooperative ambient power harvester collecting ubiquitous radio frequency and kinetic energy," IEEE Trans. Microw. Theory Techn., in early access, Sep. 2018.

[24] V. Palazzi, M. del Prete, and M. Fantuzzi, "Scavenging for Energy: A Rectenna Design for Wireless Energy Harvesting in UHF Mobile
Telephony Bands," IEEE Microw. Mag., vol. 18, no. 1, pp. 91-99, Jan.Feb. 2017.

[25] H. Sun, Y. X. Guo, M. He, and Z. Zhong, "Design of a high-efficiency $2.45-\mathrm{GHz}$ rectenna for low-input-power energy harvesting," IEEE Antennas Wireless Propag. Lett., vol. 11, pp. 929-932, 2012.

[26] M. Zeng, A. S. Andrenko, X. Liu, Z. Li, and H.-Z. Tan, "A compact fractal loop rectenna for RF energy harvesting," IEEE Antennas Wirel. Propag. Lett., vol.16., pp. $2424-2427,2017$.

[27] J. A. Hagerty, F. B. Helmbrecht, W. H. McCalpin, R. Zane, and Z. B. Popović, "Recycling ambient microwave energy with broad-band rectenna arrays," IEEE Trans. Microw. Theory Techn., vol. 52, no. 3, pp. 1014-1024, Mar. 2004.

[28] C. R. Valenta and G. D. Durgin, "Harvesting wireless power: Survey of energy-harvester conversion efficiency in far-field, wireless power transfer systems," IEEE Microw. Mag., vol. 15, no. 4, pp. 108-120, Jun. 2014.

[29] N. Shariati, W. ST Rowe, J. R. Scott, and K. Ghorbani, "Multi-service highly sensitive rectifier for enhanced RF energy scavenging." Sci. Rep., vol. 5, 9655, May 2015

[30] C. Song, et al., "A novel six-band dual CP rectenna using improved impedance matching technique for ambient RF energy harvesting," IEEE Trans. Antennas Propag., vol. 64, no. 7, pp. 3160-3171, Jul. 2016.

[31] V. Palazzi, et al., "A novel ultra-lightweight multiband rectenna on paper for RF energy harvesting in the next generation LTE bands," IEEE Trans. Microw. Theory Techn., vol. 66, no. 1, pp. 366-379, Jan. 2018.

[32] H. Sun and G. Wen, "A new rectenna using beamwidth-enhanced antenna array for RF power harvesting applications," IEEE Antennas Wirel. Propag. Lett., vol.16, pp. 1451 - 1454, 2017.

[33] M. Mattsson, C. I. Kolitsidas, and B. L. G. Jonsson, "Dual-band dualpolarized full-wave rectenna based on differential field sampling," IEEE Antennas Wirel. Propag. Lett., vol. 17, no. 6, pp. 956 - 959, Jun. 2018.

[34] C. Song, Y. Huang, P. Carter, J. Zhou, S. D. Joseph, and G. Li, "Novel compact and broadband frequency-selectable rectennas for a wide inputpower and load impedance range," IEEE Trans.Antennas Propag., vol. 66 , no. 7, pp. 3306 - 3316, Jul. 2018

[35] A. Massa, G. Oliveri, F. Viani, and P. Rocca, "Array designs for longdistance wireless power transmission: State-of-the-art and innovative solutions," Proc. IEEE, vol. 101, no. 6, pp. 1464 - 1481, Jun. 2013.

[36] S. Shen, C.-Y. Chiu, and R. D. Murch, "Multiport pixel rectenna for ambient RF energy harvesting," IEEE Trans. Antennas Propag., vol. 66, no. 2, pp. 644-656, Feb. 2018.

[37] S.-T. Khang, D.-J. Lee, I.-J. Hwang, T.-D. Yeo, and J.-W. Yu, "Microwave power transfer with optimal number of rectenna arrays for midrange applications," IEEE Antennas Wirel. Propag. Lett., vol. 17, no. 1, pp. 155 - 159, Jan. 2018

[38] Y. Yang, J. Li, L. Li, Y. Liu, B. Zhang, H. Zhu, K. Huang, “A 5.8 GHz circularly polarized rectenna with harmonic suppression and rectenna array for wireless power transfer," IEEE Antennas Wirel. Propag. Lett., vol. 17, no. 7, pp. 1276 - 1280, Jul. 2018.

[39] T. S. Almoneef, F. Erkmen, M. A. Alotaibi, and O. M. Ramahi, "A new approach to microwave rectennas using tightly coupled antennas," IEEE Trans.Antennas Propag., vol. 66, no. 4, pp. 1714 - 1724, Apr. 2018

[40] M. C. Tang, H. Wang and R. W. Ziolkowski, "Design and testing of simple, electrically small, low-profile, Huygens source antennas with broadside radiation performance," IEEE Trans. Antennas Propag., vol. 64, no. 11 , pp. 4607-4617, Nov. 2016

[41] W. Lin and R. W. Ziolkowski, "Electrically-small, low-profile, Huygens circularly polarized antenna," IEEE Trans. Antennas Propag., vol. 66, no. 2, pp. 636-643, Feb. 2018.

[42] C. A. Balanis, 3rd Ed., Antenna Theory. New York: John Wiley \& Sons, 2005.

[43] R. W. Ziolkowski, "Huygens multipole arrays to realize unidirectional needle-like radiation," Phys. Rev. X, vol. 7, 031017, Jul. 2017.

[44] Z. X. Du and X. Y. Zhang, "High-efficiency single- and dual-band rectifiers using a complex impedance compression network for wireless power transfer," IEEE Trans. Industrial Electron., vol. 65, no. 6, pp. 5012-5022, June 2018.

[45] Q. W. Lin and X. Y. Zhang, "Differential rectifier using resistance compression network for improving efficiency over extended input power range," IEEE Trans. Microw. Theory Techn., vol. 64, no. 9, pp. 29432954, Sep. 2016

[46] N. Zhu, R. W. Ziolkowski, and H. Xin, "A metamaterial-inspired, electrically small rectenna for high-efficiency low power harvesting and scavenging at the GPS L1 frequency," Appl. Phys. Lett., vol. 99, 114101, Sep. 2011. 
[47] C. Song, Y. Huang, J. Zhou, P. Carter, S. Yuan, Q. Xu, and Z. Fei, "Matching network elimination in broadband rectennas for highefficiency wireless power transfer and energy harvesting," IEEE Trans. Industrial Electron., vol. 64, no. 5, pp. 3950-3961, May 2017.

[48] W. Lin, R. W. Ziolkowski, and T. C. Baum, " $28 \mathrm{GHz}$ compact omnidirectional circularly polarized antenna for Device-to-Device communications in the future 5G systems," IEEE Trans. Antennas Propag., vol. 65, No. 12, pp. 6904-6914, Dec. 2017.

[49] Data Sheet of HSMS-286x Series Schottky Diodes, Broadcom Limited, Application Note [Online]. Available: https://www.broadcom.com.

[50] W. C. Brown, "The Design of Large Scale Terrestrial Rectennas for LowCost Production and Erection", Proc. of IEEE GMIT Int. Microwave Symposium, pp.349-351, 1978

[51] J. O. McSpadden, L. Fan, and K. Chang, "Design and experiments of a high conversion efficiency $5.8 \mathrm{GHz}$ rectenna", IEEE Trans. Microw. Theory Techn., vol.46, no.12, pp.2053- 2060, Dec. 1998.

[52] Coaxial high power amplifier ZHL-20W-13+ Datasheet. [Online] Available: https://www.minicircuits.com/pdfs/ZHL-20W-13+.pdf, Jun. 2018

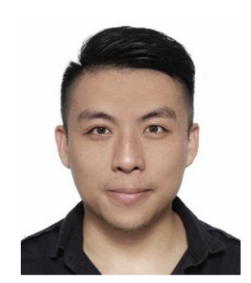

Wei LIN received his $\mathrm{PhD}$ degree in Electronic Engineering from City University of Hong Kong, Hong Kong SAR in August 2016. He received the Master an Bachelor degrees, both in Electronic Engineering, at the South China University of Technology, Guangzhou, China in July 2012 and July 2009, respectively. He worked as a Research Associate at the Nanyang Technological University, Singapore from August 2012 to August 2013 and as a Postdoc Research Associate at University of Technology Sydney, Australia from October 2016 to September 2018.

Dr. Lin is currently a Chancellor's Postdoctoral Research Fellow with the Global Big Data Technologies Centre, School of Electrical and Data Engineering, University of Technology Sydney, Ultimo NSW, Australia. Dr. Lin received many academic awards, which mainly include the Best Paper Award (First Prize) at the International Symposium on Antennas and Propagation (ISAP 2018); the Best Young Professional Paper Award (First prize) at the 3rd Australian Microwave Symposium (AMS2018); the Best Poster Paper Award at the 2nd international conference on Electromagnetic Materials and Technologies for the Future (EM-MTF2017); a Talent Development Scholarship from the Hong Kong Government; and the Young Scientist Award at the IEEE Region 10 conference (TENCON2015). Dr. Lin was the recipient of the 2018 UTS Early Career Researcher Grant Award and 2019 UTS Chancellor's Postdoctoral Research Fellowship. He serves as reviewers for several IEEE and IET journals and received the Outstanding Reviewer Award for IEEE Antennas and Wireless Propagation Letters in July 2018.

His research interests include the designs of circularly polarized antennas, electrically small antennas, reconfigurable antennas, HF antennas, satellite antennas, millimeter wave antennas, wireless power transfer, terahertz devices, and their applications.

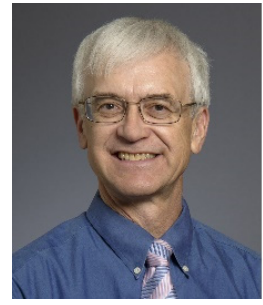

Richard W. Ziolkowski received the B.Sc. (magna cum laude) degree (Hons.) in physics from Brown University, Providence, RI, USA, in 1974; the M.S. and Ph.D. degrees in physics from the University of Illinois at Urbana-Champaign, Urbana, IL, USA, in 1975 and 1980, respectively; and the Honorary Doctorate degree from the Technical University of Denmark, Kongens Lyngby, Denmark in 2012.

$\mathrm{He}$ is currently a Distinguished Professor in the Global Big Data Technologies Centre in the Faculty of Engineering and Information Technologies (FEIT) at the University of Technology Sydney, Ultimo NSW, Australia. He became a Professor Emeritus at the University of Arizona in 2018, where he was a Litton Industries John M. Leonis Distinguished Professor in the Department of Electrical and Computer Engineering in the College of Engineering and was also a Professor in the College of Optical Sciences. He was the Computational Electronics and Electromagnetics Thrust Area Leader with the Lawrence Livermore National Laboratory, Engineering Research Division, in Livermore, CA before joining The University of Arizona, Tucson, AZ, USA, in 1990.

Prof. Ziolkowski is the recipient of the 2019 IEEE Electromagnetics Award (IEEE Field Award). He is a Fellow of the Optical Society of America (OSA, 2006), and of the American Physical Society (APS, 2016). He was the Australian DSTO Fulbright Distinguished Chair in Advanced Science and Technology from 2014-2015. He was a 2014 Thomas-Reuters Highly Cited Researcher. He served as the President of the IEEE Antennas and Propagation Society in 2005 . He is also actively involved with the URSI, OSA and SPIE professional societies.

His current research interests include the application of new mathematical and numerical methods to linear and nonlinear problems dealing with the interaction of electromagnetic and acoustic waves with complex linear and nonlinear media, as well as metamaterials, metamaterial-inspired structures, nano-structures, and other classical and quantum applications-specific configurations.

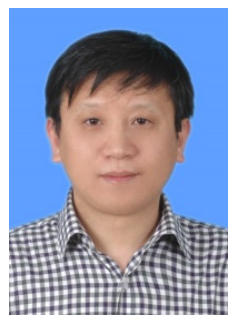

Jianquan HUANG received his M.S. in Hunan Normal University, Changsha, Hunan and Ph.D. in South China University of Technology, Guangzhou, Guangdong, China. He is working as Associate professor with Xiangnan University, Chenzhou, China from December 2012. $\mathrm{He}$ is also directing the Microwave Communication Joint Lab of Xiangnan University and Gospell. Dr Huang was a visiting scholar at the Global Big Data Technologies Centre, University of Technology Sydney, Australia.

His research interests are mainly in the fields of circularly polarized planar antenna and array, reconfigurable planar antenna. 